\title{
Percepção Discente frente às Práticas Sustentáveis de Microbiologia no Ensino Fundamental
}

\section{Insight Students face to Microbiology Practices in Sustainable Elementary School}

\section{Ana Léa de Oliveira Araújo"; Karine Silva Pimentel² ; Jonas Felipe da Silva Araújo ${ }^{3}$; Bruno Edson Chaves ${ }^{4}$, Lydia Dayanne Maia Pantoja ${ }^{5}$}

1 Bióloga pela Universidade Estadual do Ceará, Fortaleza, Ceará, Brasil analea.araujo@ aluno.uece.br 2 Bióloga pela Universidade Estadual do Ceará, Fortaleza, Ceará, Brasil karine3pimentel@gmail.com 3 Biólogo pela Universidade Estadual do Ceará, Fortaleza, Ceará, Brasil jonas.felipe@ aluno.uece.br

4 Doutorando em Ciências Biológicas (Botânica) pelo programa de pós-graduação em Botânica da Universidade de São Paulo - USP e docente da Universidade Estadual do Ceará, Fortaleza, Ceará, Brasil bruno.edson@uece.br/ORCID 00000003-1678-1393

5 Doutora em Engenharia Civil (Saneamento Ambiental) e docente da Universidade Estadual do Ceará, Fortaleza, Ceará, Brasil lydia.pantoja@uece.br/ORCID 0000-0002-4446-7230

\section{Recebido em Abril/2018. Publicado em Abril/2020}

Palavras-chave:

Aprendizagem. Microbiologia. Aulas práticas. Materiais sustentáveis. Ensino de Ciências.

\section{Keywords:}

Learning. Microbiology. Practical classes. Sustainable materials. Science teaching.
RESUMO: O ensino de Microbiologia possui algumas dificuldades devido às características peculiares apresentadas pelos seres microscópicos, sendo necessário o professor buscar novos métodos e ferramentas para expor esse conteúdo em sala de aula. Para estudo do mundo microbiológico é preciso à utilização de estratégias e ferramentas didáticas que possam facilitar o processo de ensino e aprendizagem. Nesse ínterim, o presente trabalho objetivou elaborar e aplicar quatro aulas práticas em Microbiologia com a utilização de materiais alternativos e de fácil obtenção para alunos do $7^{\circ}$ ano do Ensino Fundamental do município Fortaleza-CE e analisar através de questionário a percepção dos discentes sobre as aulas práticas realizadas. Foram elaborados quatro planos de aulas com base na literatura atual em Microbiologia e um questionário com base na escala de Linkert. Este trabalho mostrou que $80 \%$ dos alunos pesquisados possuem conhecimentos sobre os microorganismos, 88,4\% dos discentes participantes da pesquisa consideram que a realização de aulas práticas é muito importante para o aprendizado em Ciências quando o conteúdo estudado se refere a Microbiologia e $75 \%$ dos discentes afirmam que a busca de materiais alternativos para a realização de aulas práticas contribuiria para que essas aulas ocorressem com maior frequência. Conclui-se que os alunos consideram a aula prática essencial para seu aprendizado e importante o uso de materiais alternativos no processo ensino e aprendizagem em Microbiologia, além de mostrar a necessidade do uso de atividades que sejam dinâmicas e atraentes para os alunos.

ABSTRACT: Teaching of Microbiology has some difficulties due to the peculiar characteristics presented by microscopic beings, the teacher is necessary to seek new methods and tools to expose this content in the classroom. To study of microbial world we need to use strategies and teaching tools that can facilitate the process of teaching and learning. Meanwhile, this paper aims to develop and implement four practical lessons in microbiology with the use of alternative materials and easily accessible for students from the 7th grade of elementary school of Fortaleza municipality and analyze through a questionnaire the perception of students on classes held practices. four lesson plans were developed based on the current literature in Microbiology and a questionnaire based on the scale of Linkert. This 
study showed that $80 \%$ of students have knowledge of micro-organisms, $88.4 \%$ of survey participants believe that students conducting practical classes is very important for learning in science when the content studied refers to microbiology and $75 \%$ of students say that the search for alternative materials for conducting practical classes contribute to these classes occur more frequently. It is concluded that students consider the essential practical class for their learning and important use of alternative materials in the teaching and learning in microbiology, as well as showing the need to use activities that are dynamic and attractive for students.

\section{INTRODUÇÃO}

A Microbiologia é a ciência que estuda o papel dos micro-organismos no mundo, principalmente em relação à sociedade humana, ao corpo humano e ao meio ambiente. Assim, ela pode abordar temas com aspectos de natureza básica e prática podendo ser conceituada como fonte de produtos e processos importantes que trazem benefícios para humanidade.

Uma vez que estes organismos são muito pequenos, as atividades práticas fundamentais para compreendê-la e interpretar o conteúdo, além de desenvolver a capacidade de observar e de analisar dados. Contudo, os preços de vidrarias, meios de cultura e outros equipamentos são bastante elevados o que torna difícil para muitas escolas comprar materiais e manter laboratórios na área de Ciências o que inviabiliza a realização de aulas práticas (BARBOSA; BARBOSA, 2010).

Cassanti et al. (2008) afirmam que é pertinente o desenvolvimento de atividades de simples execução e baixo custo para as escolas do Brasil, tentado amenizar possíveis problemas relacionados à falta de tempo do professor para organizar aulas mais complexas (como alguns tipos de experimentos práticos), bem como a possível falta de recursos financeiros. Além de que, esse é um problema não apenas de escolas públicas, mas também de muitas escolas particulares de pequeno porte localizadas em Fortaleza.

Tomando como base está problemática, o presente trabalho buscou avaliar a percepção dos alunos de Ensino Fundamental em aulas práticas de Microbiologia com uso de materiais alternativos; bem como a visão dos mesmos sobre a busca por materiais sustentáveis para a realização de tais atividades.

\section{METODOLOGIA}

O presente trabalho é de caráter quali-quantitativo e utilizou técnicas padronizadas de coleta de dados: observação sistemática e questionário; este último, segundo Ludke e André (2001), pode ser um instrumento valioso na pesquisa qualitativa.

A pesquisa foi realizada em uma escola de educação básica particular localizada no município de Fortaleza, Ceará. Participaram da mesma, 52 alunos (30 do turno manhã e 22 do turno tarde) entre doze e treze anos regularmente matriculados no $7^{\circ}$ ano do Ensino 
Fundamental II; esta série foi escolhida, pois é neste período que os alunos veem conteúdos ligados à biodiversidade dos micro-organismos.

Foram realizadas quatro aulas práticas no laboratório de ciências, tomando com base os experimentos sugeridos em livros indicados pelo Plano Nacional do Livro Didático-PNLD (MEC, 2015), bem como, por serem práticas de fácil execução, com uso de material alternativo/reciclável e com resultados visualizados no momento do próprio procedimento ou no máximo em 24 horas, conforme mostra a Tabela 1.

Tabela 1 - Aulas práticas realizadas com alunos do $7^{\circ}$ ano do Ensino Fundamental do município Fortaleza-CE.

\begin{tabular}{|c|c|c|c|}
\hline Aula & Título da aula prática & $\begin{array}{c}\text { Tempo para } \\
\text { visualização do } \\
\text { resultado da prática }\end{array}$ & Referência \\
\hline $1^{\mathrm{a}}$ & $\begin{array}{c}\text { Visualização do crescimento microbiano } \\
\text { com a utilização de materiais } \\
\text { alternativos. }\end{array}$ & $24 \mathrm{~h}$ & $\begin{array}{l}\text { Linhares; Gewandsznajder } \\
\text { (2010) }\end{array}$ \\
\hline $2^{\mathrm{a}}$ & $\begin{array}{c}\text { Detectando a presença de micro- } \\
\text { organismos na água com uso de } \\
\text { microscópio caseiro. }\end{array}$ & Imediato & César; Sezar; Caldini (2011) \\
\hline $3^{\mathrm{a}}$ & $\begin{array}{l}\text { Eficácia da lavagem das mãos na } \\
\text { redução dos micro-organismos da pele. }\end{array}$ & $24 \mathrm{~h}$ & César; Sezar; Caldini (2011) \\
\hline $4^{\mathrm{a}}$ & $\begin{array}{l}\text { Testando a eficácia de produtos naturais } \\
\text { contra crescimento microbiano. }\end{array}$ & $24 \mathrm{~h}$ & Amabis; Martho (2013) \\
\hline
\end{tabular}

Fonte: os autores.

Para a execução da primeira aula prática foram escolhidos dois alunos para realizar coletas de material, um aluno fez coleta da maçaneta do banheiro e outro fez coleta de material do vaso sanitário (Figura 1), o objetivo da aula era analisar em qual local apresentaria a maior contaminação. Para execução da segunda aula prática dois discentes foram escolhidos, sendo que um coletou a água da pia do banheiro e outro a água do bebedouro da sala dos professores (Figura 2), o objetivo da aula era comparar qual água apresentava mais formas microscópicas.

Figura 1 - Aplicação da primeira aula prática, visualização do crescimento microbiano com a utilização de materiais alternativos. 
Ensino, Saúde e Ambiente - V13 (1), pp. 20-30, Abr. 2020

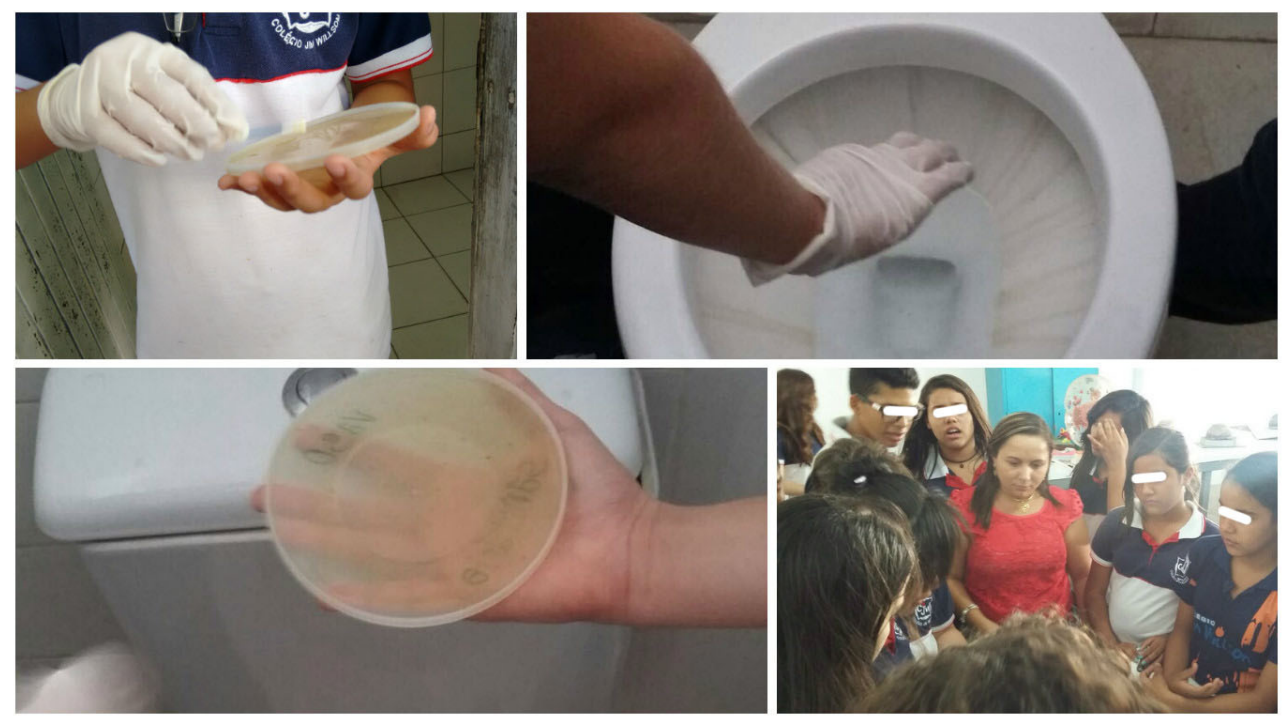

Fonte: os autores.

Figura 2 - Execução da segunda aula prática, detectando a presença de micro-organismos na água com uso de microscópio caseiro.

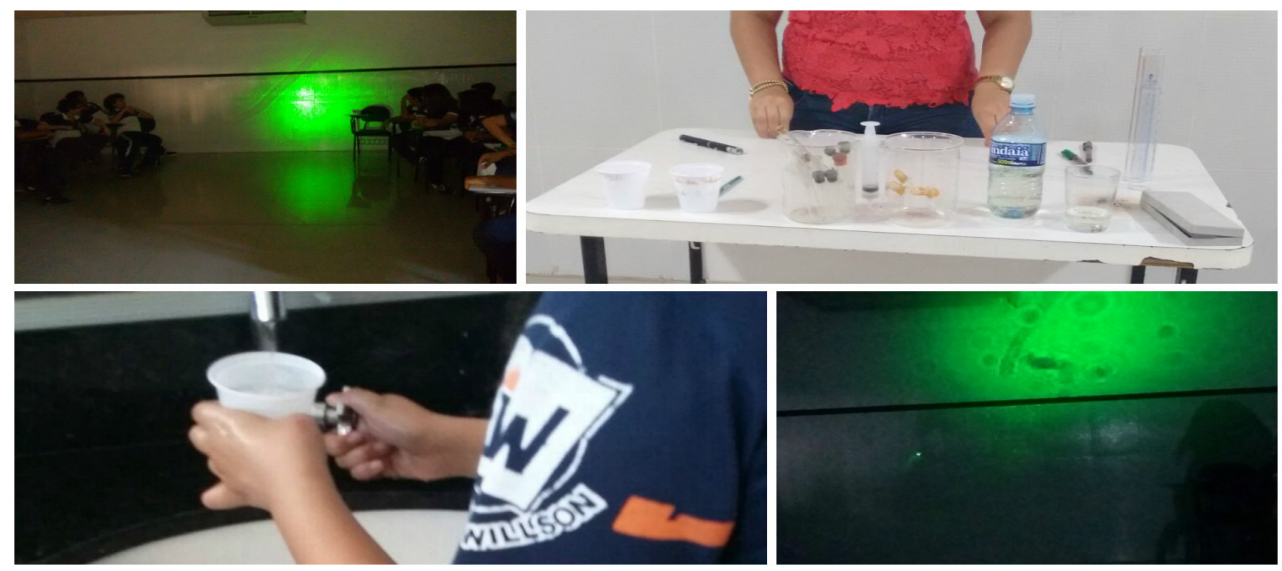

Fonte: os autores.

A terceira prática teve como objetivo verificar a eficácia da lavagem das mãos para reduzir os micro-organismos, para tanto, quatro alunos (dois que lavaram a mão com água, sabão e, posteriormente, álcool; e dois com mãos sujas) foram escolhidos para semearem o meio de cultura (Figura 3).

Por fim, para execução da quarta aula prática (Figura 4), que objetivou testar a eficácia de produtos naturais contra crescimento microbiano. Para tanto, os alunos foram separados em quatro equipes. Cada equipe recebeu uma lamparina, três cotonetes, uma tampa com meio de cultura (simulando placas de Petri) com a presença de micro-organismos (placas usadas na primeira aula prática) e amostras de mel, alho e gengibre. Em seguida, foram orientados a dividir o meio de cultura em três espaços e semear com ajuda do cotonete em 
cada espaço um dos produtos, logo, em uma placa foi possível verificar o resultado dos três produtos.

A escolha dos alunos, por turma, em todas as aulas práticas foi realizada aleatoriamente.

Figura 3 - Momentos da terceira prática: eficácia da lavagem das mãos na redução dos micro-organismos da pele.

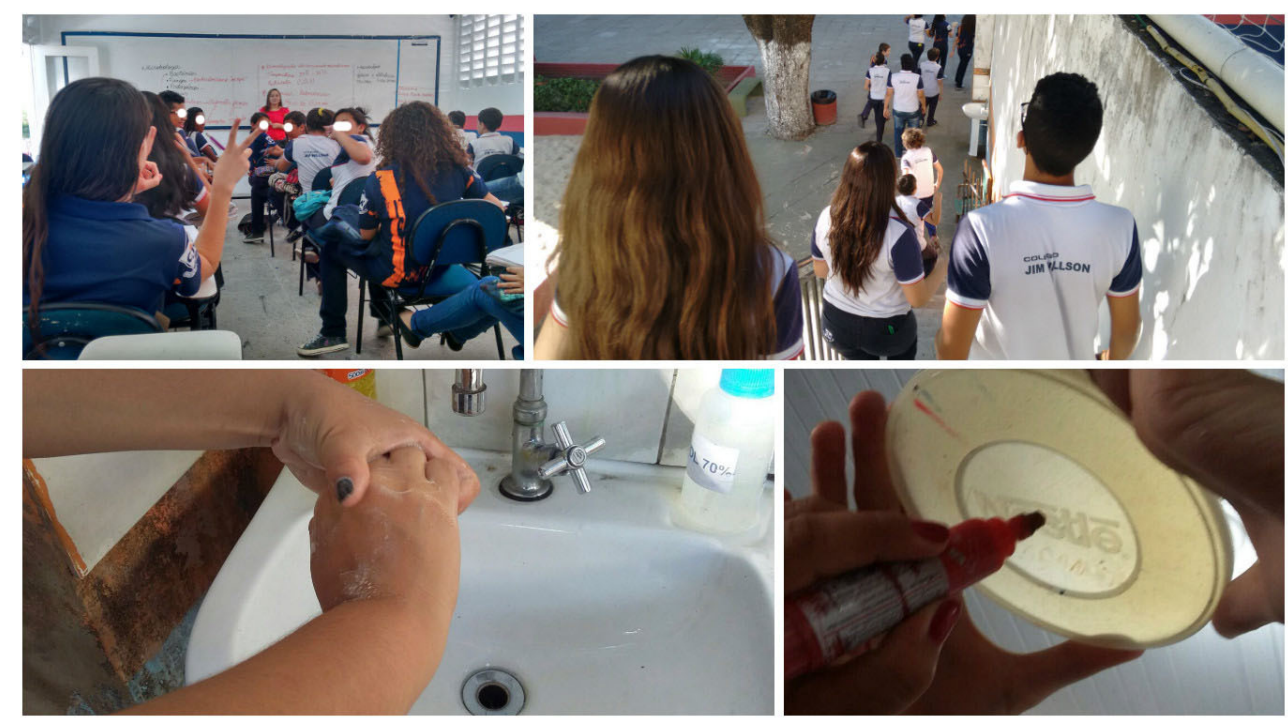

Fonte: os autores.

Figura 4 - Utilização da estufa para a prática quatro: testando a eficácia de produtos naturais contra crescimento microbiano.

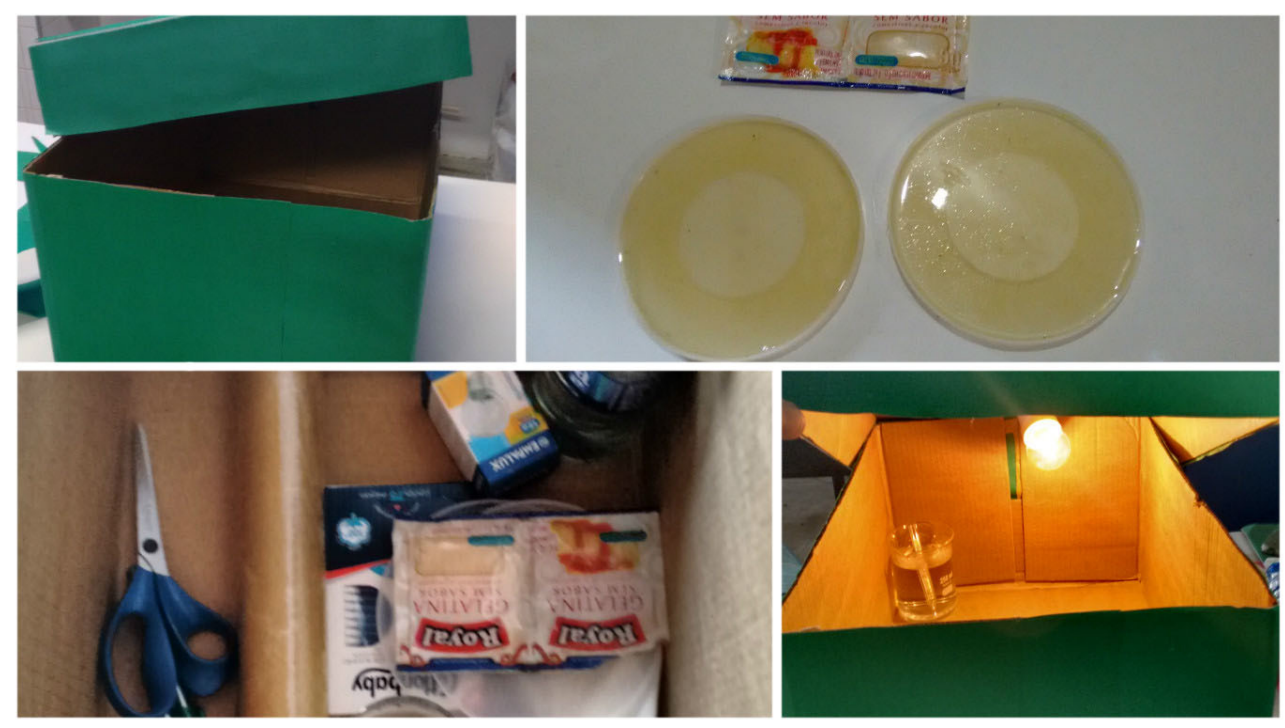

Fonte: os autores.

Posteriormente às aulas e após assinaram um Termo de Consentimento Livre e Esclarecido (TCLE), esses alunos foram convidados a responder o questionário sobre a atividade realizada, de forma a analisar a percepção dos discentes sobre o respectivo assunto. 
O questionário foi elaborado com base na escala de Likert, que de acordo com Cunha (2007) é composta por um conjunto de frases (itens) em relação a cada uma das quais se pede ao sujeito que está a ser avaliado para manifestar o grau de concordância desde o discordo totalmente (nível 1), até ao concordo totalmente (nível 5).

Vale ressaltar que antes das atividades um termo de assentimento foi encaminhado para os pais destes alunos a fim dos mesmos também concordarem com a participação dos filhos na pesquisa.

\section{RESULTADOS E DISCUSSÃO}

Quanto à execução das aulas práticas foi observado que, durante as aulas, os alunos mostraram-se bem agitados, tendo sido difícil a execução das práticas em alguns momentos, especialmente durante a primeira aula prática. Entretanto, os mesmos se mostram bastante entusiasmados, principalmente durante a segunda aula prática (microscópio caseiro), pois todos os alunos queriam testar alguma amostra. A $3^{\mathrm{a}}$ e $4^{\mathrm{a}}$ aulas práticas foram as que tiveram maior participação dos alunos, tendo eles realizados com sucesso todos os procedimentos da aula estipulados no plano de aula.

O plano de aula é certamente um detalhamento do plano de ensino, é uma especificação do mesmo. O detalhamento da aula é fundamental para obtermos uma qualidade no ensino, sendo assim o plano de aula torna-se indispensável (LIBÂNEO, 1994). Moretto (2007) também afirma que planejar é organizar ações. Essa é uma definição simples, mas que mostra uma dimensão da importância do ato de planejar, uma vez que o planejamento deve existir para facilitar o trabalho tanto do professor como do aluno. O planejamento deve ser uma organização das ideias e informações.

Frente ao comportamento dos alunos, constatou-se que a maior dificuldade, em especial, no que tange ao comportamento, foi observada na primeira aula, isso se deve em especial pelo anseio do aluno junto ao novo, estresse que acontece no cotidiano acadêmico (MOREIRA, 2014), a partir da segunda aula em diante, os alunos já se mostraram bem mais receptivos as atividades.

No que se refere à percepção discente das atividades práticas notou-se, com base nos questionários, que $80 \%$ dos alunos afirmaram que o conhecimento de microbiologia transmitido nas aulas práticas está presente nos capítulos de vírus, bactérias e fungos do livro didático. Este dado mostra que os alunos apresentam conhecimento sobre quem são os microorganismos, sendo as aulas práticas um reforço neste aprendizado. Em trabalho, sobre vídeos didáticos para ensino de Microbiologia no Ensino Fundamental, desenvolvido por Barbosa e 
Oliveira (2015) 87,5\% dos discentes também afirmaram saber sobre exemplos de microorganismos.

Os demais alunos (20\%) discordaram da afirmação. Cassanti et al. (2008) afirmam que o mundo microbiológico pode ser extremamente abstrato para alguns alunos, pois embora seja parte do dia a dia não se pode percebê-los de forma mais direta por meio dos sentidos

Com o objetivo analisar a percepção da importância das aulas práticas no ensino de ciências, $88,4 \%$ dos alunos concordaram que as aulas práticas são importantes para o ensino de ciências. Tal resultado está de acordo com Giordan (1999) uma vez que este trabalho afirma que a experimentação pode despertar um forte interesse entre os alunos em diversos níveis de escolarização.

A maioria dos alunos (75\%) acredita que se o professor buscasse materiais alternativos para realização de aulas práticas, essas aulas ocorreriam com maior frequência. Outros 25\% discordaram parcialmente com essa afirmativa. Possobom, Okada e Diniz (2003), também analisando a visão do discente, mostram que apesar das precárias condições apresentadas em escolas da rede paulista com relação a materiais e espaço para atividades de laboratório é possível contornar todos os problemas ou sua maioria, com um pouco de esforço e com a adaptação de ambientes e utilização de materiais simples com baixo custo, proporcionando assim, um aprendizado mais eficiente e mais motivador que as tradicionais aulas meramente expositivas.

Dentre os materiais importantes para uso em práticas laboratoriais de microbiologia encontra-se o Ágar Batata, importante meio de cultura para observar crescimento microbiano (SIDRIM; ROCHA, 2004). Quando questionado sobre a utilização deste material no crescimento microbiano a turma ficou confusa, uma vez que o percentual das nos itens foram muito próximas: 19,2\% concordaram totalmente, $23 \%$ concordaram parcialmente, $26,9 \%$ discordaram parcialmente e 32,6\% discordaram totalmente. A hipótese levantada é que os alunos ficaram indecisos nessa questão devido ao pouco convívio com materiais utilizado para este fim, tendo pouco conhecimento sobre o funcionamento de um laboratório para estudos microbiológicos.

Os alunos foram analisados sobre a utilização de materiais simples para visualização do crescimento microbiano, nessa perspectiva, 65,3\% dos alunos concordaram que foi bastante proveitoso a utilização desses materiais. Este dado está de acordo com os apresentados por Cassanti et al. (2008) onde ele afirma que as técnicas básicas de cultivo de colônias de micro-organismos em meio sólido mostraram-se satisfatórias.

Embora seja necessária a utilização de estufas para verificar o crescimento microbiano, após as práticas $78,8 \%$ dos discentes concordaram totalmente que a mesma pode 
ser facilmente substituída por uma de baixo custo, como a estufa papelão produzida. Os resultados superam os de Crisóstomo et al. (2014), os autores citam que a elaboração de matérias de laboratório de baixo custo também obteve aprovação de 45,4\% dos alunos participantes da pesquisa.

Quando questionados sobre a importância de visualizar o crescimento microbiano para melhor aprendizagem em Microbiologia, 65,3\% dos alunos concordam totalmente da importância dessa visualização e $25 \%$ concordaram parcialmente, os demais não concordam. Este dado corrobora com o trabalho de Conceição et al. (2014), os autores relatam que o cultivo de micro-organismo proporcionou a participação e envolvimento dos discentes, bem como despertou a curiosidade dos estudantes que participaram diretamente do projeto, estimulou o interesse pelo o conteúdo e ajudou a relacionar a teoria com a prática, contribuindo para o entendimento dos índices e riscos de contaminação através de microorganismos no ambiente escolar.

Por fim, ao serem questionados sobre a visualização de micro-organismos apenas com o uso de microscópio óptico, 36,5\% dos alunos, discordaram totalmente e $25 \%$ dos alunos concordaram, acredita-se que esses dados sejam devido ao experimento de microbiota da água realizado, onde os alunos coletaram água de diferentes locais da escola e ao utilizarem o microscópio caseiro visualizaram algumas formas microscópicas, através dessa prática os alunos passaram de fato a ver o mundo microbiano, tornando o conteúdo mais próximo deles. De acordo com Silva et al. (2009) foi constatado que aulas práticas, experiências simples e uso de microscópio, há maior participação, interesse e entusiasmo dos alunos. As aulas práticas que utilizam tal instrumento como recursos permitem a melhor abrangência do conteúdo, aproximando o discente da realidade, e assim ele pode que em biologia é muito mais interessante de que somente teoria.

Ainda buscou-se analisar o conhecimento dos discentes sobre a microbiota humana, quanto a isto, verificou-se que $46,1 \%$ concordaram totalmente que o corpo humano possui uma carga de bactérias e fungos seguidos de 40,3\% que concordaram parcialmente com a afirmativa. Os dados corroboram como Cassanti et al. (2008) e Barbosa e Oliveira (2015), o primeiro afirma que praticamente todos os alunos possuíam a informação de que há bactérias em todos os lugares, enquanto o segundo relata que quando questionados sobre a presença de micro-organismos em suas mãos.

Quando questionados sobre o ato de lavar as mãos ajuda evitar doenças causadas por micro-organismos, 94,2\% concordaram totalmente, o que mostra que os alunos associam os micro-organismos como causadores de doenças. Dados semelhantes foram constatados na literatura, por exemplo, Bizerra et al. (2009) relatam que $71 \%$ das crianças entrevistadas em 
sua pesquisa associam micro-organismos a doenças ou características negativas. E por isso o ato de lavar as mãos seria uma medida primária de combate as doenças.

Nos últimos questionamentos sobre a aula prática de produtos naturais contra microorganismos, os alunos mostraram concordância de que esse tipo de aula auxilia na compreensão da ação dos antimicrobianos, uma vez que os produtos agem inibindo crescimento de micro-organismos, o que corrobora a literatura que afirma que o uso de produtos naturais com poder curativo (NUÑEZ; CIOSAK, 2003).

\section{CONCLUSÃO}

Notou-se que os alunos consideram aulas práticas como essenciais para seu aprendizado e que tais práticas pode ser uma ferramenta para a prevenção e combate de algumas doenças. Ressalta-se ainda a importância do uso de materiais alternativos e sustentáveis no processo de ensino e aprendizagem em Microbiologia.

Aulas mais dinâmicas e atraentes não é uma necessidade somente da escola investigada, mas de todas as escolas brasileiras. Para tanto, é necessário que os docentes produzam e desenvolvam materiais alternativos que ajudem a transformar cada vez mais o processo de ensino e aprendizagem convencional; de forma que iniciativas como esta sejam cada vez mais aprimoradas, buscando auxiliar na aprendizagem da Microbiologia no Ensino Fundamental.

\section{REFERÊNCIAS}

AMABIS, J. M.; MARTHO, G. R. Biologia em Contexto: a diversidade dos Seres Vivos. v. 3. São Paulo, Editora Moderna, 2013.

BARBOSA, F. H. F.; BARBOSA, L. P. J. L. Alternativas metodológicas em Microbiologia viabilizando atividades práticas. Revista de Biologia e Ciências da Terra, v. 10, n. 2, p. 134$143,2010$.

BARBOSA, G. F; OLIVEIRA, C. N. Estratégias para o Ensino de Microbiologia: uma Experiência com Alunos do Ensino Fundamental em uma Escola de Anápolis-GO. Cient.

Ciênc. Human. Educ., Londrina, v. 16, n. 1, p. 5-13, 2015.

BIZERRA, A.; DOMINGUES, C.; INGLEZ, G. C.; GONÇALVES, V. M.; IMPARATO, B. A.; HENRIQUE, B. C.; PEREIRA, F. F.; VIEIRA, J. L. A.; CASADEI, K.; LEPORO, N.; FRANCO, M. et al. Crianças pequenas e seus conhecimentos sobre microrganismos. In: Encontro Nacional de Pesquisa em Educação em Ciências, 7, Florianópolis, 2009, Florianópolis. Anais... Florianópolis: ENPEC, 2009.

CAPELETTO, A. Biologia e Educação ambiental: roteiros de trabalho. Editora Ática, 1992. p. 224.

CASSANTI, A. C.; CASSANTI, A. C.; ARAUJO, E. E. de; URSI, S. Microbiologia

Democrática: estratégias de ensino-aprendizagem e formação de professores. Colégio Dante Alighieri: São Paulo, 2008.

CÉSAR, S.J.; SEZAR, S.; CALDINI, J.N. Biologia. 9. ed. São Paulo: Saraiva. 2011. 
CONCEIÇÃO, B. C.; ROCHA, B. R. J.; SANTOS, C.W.; CÂMARA, J. T. Trabalhando microrganismos através de projetos didáticos no $2^{\circ}$ ano do ensino médio no centro de ensino Inácio Passarinho - CEIP, Caxias, Maranhão, Brasil. Departamento de Química e Biologia do Centro de Estudos Superiores de Caxias, da Universidade Estadual do Maranhão (CESC/UEMA). 2014.

CRISOSTOMO, L. C. S.; FEREIRA, M. P.; MARINHO, M. M.; CASTRO, R. R.;

MARINHO, E. S. A utilização de materiais alternativos como proposta para o ensino de Química, na perspectiva dos alunos do curso de Licenciatura em Química. XII Simpósio Brasileiro de Educação Química. Anais... Fortaleza, 2014.

CUNHA, A. M. L. Modelos Rasch e Escalas de Likert e Thurstone na medição de atitudes. Universidade de Lisboa Faculdade de Ciências Departamento de Estatística e Investigação Operacional. Mestrado em Probabilidades e Estatística, 2007.

GIORDAN, M. O papel da experimentação no ensino de ciências. Química nova na escola, n. 10, p. 43-49, 1999.

LIBÂNEO, C. J. Didática. Cortez, 1994. 263p.

LINHARES, S. V.; GEWANDSZNAJDER, F. Biologia Hoje. São Paulo; Ática, 2010.

LÜDKE, M; ANDRÉ, M. E. D. A. Pesquisa em educação: abordagens qualitativas. São Paulo: Epu, 2001.

MANZATO, A. J. S.; BARBOSA, A. A Elaboração de questionários na pesquisa quantitativa. Departamento de Ciência de Computação e Estatística - IBILCE - UNESP, 2014.

MOREIRA, A. E. da C. 118f. Relações entre as estratégias de ensino do professor, com as estratégias de aprendizagem e a motivação para aprender de alunos do ensino

fundamental 1. Dissertação (Mestrado em Educação), Universidade Estadual de Londrina, Centro de Educação, Comunicação e Artes, Programa de Pós-Graduação em Educação, 2014. MORETTO, V. P. Planejamento: planejando a educação para o desenvolvimento de competências. Petrópolis, RJ: Vozes, 2007.

NUÑEZ, H. M.; CIOSAK, S. I. Terapias alternativas/complementares: o saber e o fazer das enfermeiras do distrito administrativo 71 - Santo Amaro - São Paulo. Rev Enf USP, v. 37, n. 3, p. 11-18. 2003.

POSSOBOM. F.; OKADA. K. F.; DINIZ. S. E. R. Atividades práticas de laboratório no ensino de biologia e de ciências: relato de uma experiência. 2003. Disponível em:

$<$ http://www.lsgasques.blogs.unipar.br/files/2009/09/Aulas-Pr\%C3\%A1ticas-no-ensinodebiologia-e-de-Ci\%C3\%AAncias-Roteiros.pdf> Acesso em: 27 jan. 2016.

SIDRIM J. J. C., ROCHA, M. F. G. Micologia Médica à luz de autores contemporâneos. Rio de Janeiro: Guanabara Koogan, 2004.

SILVA, D. R. M.; VIEIRA, N. P.; OLIVEIRA, A. M. O ensino de biologia com aulas práticas de microscopia: uma experiência na rede estadual de Sanclerlândia- GO. Anais... III EDIPEEncontro Estadual de Didática e Prática de Ensino. p. 1-4, 2009.

\section{SOBRE OS AUTORES}

ANA LÉA DE OLIVEIRA ARAÚJO: Licenciada em Ciências Biológicas pela UECE experiência na área de entomologia com ênfase em mirmecologia e experiência em microbiologia analisando qualidade do ar de um campus universitário da UECE. Atuando como professora de Ciências e Biologia da rede particular de ensino. Coletou dados da pesquisa e escreveu o artigo.

KARINE SILVA PIMENTEL: possui graduação em Ciências Biológicas pela Universidade Estadual do Ceará (2016). Coletou dados da pesquisa e escreveu o artigo.

JONAS FELIPE DA SILVA ARAÚJO: graduado em Ciências Biológicas pela Universidade Estadual do Ceará (UECE). Atua e tem interesses nas áreas de micologia, 
microbiologia ambiental, monitoramento e análise da qualidade do ar. Coletou dados da pesquisa e escreveu o artigo.

BRUNO EDSON CHAVES: possui graduação em Ciências Biológicas (licenciatura - 2008; e bacharelado - 2010) pela UECE e mestrado em Botânica pela UnB - 2012; atualmente cursa doutorado em Ciências Biológicas (botânica) pelo programa de pós-graduação em botânica da USP. É professor de botânica (assistente nível d) pela UECE na Faculdade de Educação, Ciências e Letras de Iguatu - FECLI; atuando ainda como tutor/ formador presencial de botânica pelo curso de Ciências Biológicas UECE/UAB, na modalidade a distância. Tem experiência na área de botânica, com ênfase em anatomia vegetal de poales/poaceae, atuando em três principais linhas de pesquisa: 1) anatomia aplicada a taxonomia, 2) anatomia ecológica, 3) ontogenia de estruturas foliares. Atua ainda na área de ensino de biologia, em particular, ensino de botânica, com foco em estratégias didáticas para o ensino de botânica. Catalogou dados, analisou e escreveu o artigo.

LYDIA DAYANNE MAIA PANTOJA: doutora em Engenharia Civil (área de concentração em saneamento ambiental) pela UFC (2016); mestre em Microbiologia Médica pela UFC (2008) e graduação em Ciências Biológicas pela UECE (bacharel - 2007 e licenciatura 2006). Atua como professora assistente nível d da UECE. Docente do mestrado profissional em Ensino de Biologia em Rede Nacional/Profbio. Coordena a pesquisa do curso de Ciências Biológicas a distância - UECE/UAB. É coordenadora de área do curso pré-universitário UECEVest - biologia. Tem experiência na área de microbiologia, com ênfase em microbiologia ambiental e aplicada, atuando principalmente nos seguintes temas: aerobiologia, taxonomia fúngica, fungos anemófilos e compostos orgânicos voláteis microbianos. Bem como, desenvolve trabalhos na área de pesquisa em educação a distância e formação de professores. Catalogou dados, analisou e escreveu o artigo. 